\title{
Valeurs usuelles des métaux et métalloïdes dans le sang total et les urines par ICP-MS chez cinquante-quatre sujets décédés
}

\author{
Whole blood and urine ICP-MS metal and \\ metalloid determination : usual values found \\ in fifty-four cadavers
}

Jean-Pierre GOULLÉ ${ }^{(1) *}$, Lö̈c MAHIEU ${ }^{(1)}$, Valérie MAIGNANT ${ }^{(1)}$ Daniel BOUIGE $^{(2)}$, Élodie SAUSSEREAU ${ }^{(1)}$, Christian LACROIX ${ }^{(1)}$

(1) Laboratoire de pharmacocinétique et de toxicologie Cliniques, Groupe Hospitalier du Havre, BP 24, 76083 LE HAVRE CEDEX

(2) Laboratoire de Biochimie, Groupe Hospitalier du Havre, BP 24, 76083 LE HAVRE CEDEX

*Auteur à qui adresser la correspondance : Jean-Pierre GOULLÉ,

Laboratoire de Pharmacocinétique et de Toxicologie Cliniques, Groupe Hospitalier du Havre, BP 24, 76083 LE HAVRE CEDEX, France Tél : 0232733223 - Fax : 0232733238 - E-mail : jgoulle@ch-havre.fr Ce travail a été partiellement présenté au XIV Congrès annuel de la SFTA au Touquet.

(Reçu le 5 février 2007 ; accepté après modifications le 8 mars 2007)

\section{RÉSUMÉ}

Chez un quart des sujets pour lesquels une autopsie médicolégale est demandée, une cause toxique de décès est identifiée. La recherche des causes toxiques de la mort fait appel à des techniques chromatographiques couplées : barrette de diodes, spectrométrie de masse. Il s'agit de méthodes très performantes pour de nombreux xénobiotiques organiques (médicaments, pesticides, substances variées), mais elles ne permettent pas de mettre en évidence des poisons minéraux. Pour ces derniers, un certain nombre d'analyses spécifiques, longues et fastidieuses sont nécessaires avec au minimum les dosages des cyanures et du lithium dans le cadre de l'expertise toxicologique de référence. Parmiles investigations

\section{SUMMARY}

Toxicology is considered a major topic in the field of forensic sciences. Toxicological analysis has made significant advances using chromatographic techniques, i.e. gas and liquid chromatography coupled to mass spectrometry, or liquid chromatography with diode array detector. These methods are excellent for many organic xenobiotics (drugs, pesticides, other organic substances), but are not suitable for minerals like cyanide or lithium. The standard atomic absorption spectrophotometry techniques are tedious to use, particularly when a vast number of elements are concerned. In recent years, the ICP-MS method has been extensively developed due to new high performance equipment. A thirty- 
complémentaires, la mesure des métaux et des métallö̈des est le plus souvent effectuée par spectrométrie d'absorption atomique en mode monoélémentaire. Aussi nous proposons le dosage simultané d'une trentaine de métaux et métalloüdes dans les prélèvements autopsiques sanguins et urinaires par plasma à couplage inductif relié à un spectromètre de masse (ICP-MS) pour rechercher une exposition à l'un de ces éléments. Les mesures sont réalisées sur spectromètre X7CCT Thermo Elemental (ThermoOptek, Courtabouf, France), équipé d'un passeur d'échantillon et du logiciel PlasmaLab selon une technique déjà décrite. Trente et un éléments sont quantifiés simultanément dans du sang prélevé sur fluorure et dans les urines : $\mathrm{Li}, \mathrm{Be}, \mathrm{B}, \mathrm{Al}, \mathrm{Mn}, \mathrm{Co}, \mathrm{Ni}, \mathrm{Cu}, \mathrm{Zn}, \mathrm{Ga}$, $G e, A s, S e, B r, R b, S r, M o, P d, A g, C d, S n, S b, T e, B a, W$, $P t, H g, T l, P b, B i, U$. Le vanadium est le trente deuxième élément mesuré dans les urines. La détermination du brome est effectuée de manière semi-quantitative. Les dosages sont réalisés dans le sang total et les urines selon la méthode des ajouts dosés par dilution des échantillons en milieu acide en présence de butanol et de triton. Des valeurs fréquentes dans nos conditions de recueil sont établies chez des sujets dont le décès n'est pas imputable à l'un de ces minéraux. L'impact des contenants sur les résultats obtenus a également été évalué. La validité des résultats est garantie par la participation de notre laboratoire au contrôle de la qualité par ICP-MS dans le sang total, les urines et les cheveux, organisé par l'institut National de Santé Publique du Québec (Sainte-Foy, Canada). Les limites de détection et de quantification calculées sont excellentes et les scores obtenus au contrôle de qualité sont bons. Les valeurs fréquentes dans le sang total et les urines de 54 sujets décédés sont établies pour l'ensemble des éléments. Les applications sont nombreuses : le dosage multiélémentaire permet au cours d'une même analyse de rechercher une exposition à des poisons métallö̈diques ou métalliques $(\mathrm{As}, \mathrm{Tl})$, à des métaux lourds $(\mathrm{Pb}, \mathrm{Cd}, \mathrm{Hg})$, à des métaux variés (Al, Co, Ni, Mo, W), à des médicaments ( $\mathrm{Li}$, $\mathrm{Pt}, \mathrm{Bi}, \mathrm{Al}$ ), à des pesticides ( $\mathrm{As}, \mathrm{Pb}, \mathrm{Br}, \mathrm{Al}, \mathrm{Ba}, \mathrm{Hg}, \mathrm{Se}, \mathrm{Cu})$, à des bombes fumigènes $(\mathrm{Zn}, \mathrm{Al})$, à des halogénures $(\mathrm{Br}, \mathrm{I})$, à des lanthanides (Ce, La, Gd), ou à des uranides (Th, U). L'intérêt de la détermination du strontium et du brome dans le diagnostic de noyade en milieu contaminé par ces éléments est rappelé. La technique est également applicable à d'autres matrices biologiques : cheveux et ongles en particulier. L'ICP-MS offre ainsi des perspectives réellement nouvelles en toxicologie médico-légale. Le dosage multiélémentaire des métaux et métalloüdes devrait donc être réalisé dans tous les cas de décès inexpliqués par l'autopsie et pour lesquels l'expertise toxicologique de référence s'avère négative.

\section{MOTS-CLÉS}

ICP-MS, sujets décédés, métaux, sang total, urines.

\section{Introduction}

En France, chez un quart des sujets pour lesquels une autopsie médico-légale est demandée, une cause toxique de décès est identifiée. La recherche des causes toxiques de la mort fait classiquement appel à des techniques chromatographiques couplées : barrette de diodes, spectrométrie de masse. Il s'agit de méthodes two multi-elementary ICP-MS method is proposed for forensic blood and urine samples to detect pathologic exposure to these elements. The ICP-MS measurements were performed using a thermo Elemental X7CCT series and PlasmaLab ${ }^{\circledR}$ software. In this procedure thirty-one elements are simultaneously quantified in fluoride whole blood and urine : $\mathrm{Li}, \mathrm{Be}, \mathrm{B}$, Al, $\mathrm{Mn}, \mathrm{Co}, \mathrm{Ni}, \mathrm{Cu}, \mathrm{Zn}, \mathrm{Ga}, \mathrm{Ge}, \mathrm{As}, \mathrm{Se}, \mathrm{Br}, \mathrm{Rb}, \mathrm{Sr}, \mathrm{Mo}$, $P d, A g, C d, S n, S b, T e, B a, W, P t, H g, T l, P b, B i, U . S e m i-$ quantitative bromide analysis is also assessed and vanadium determination is added to urine. Whole blood and urine analysis were performed using a standard addition procedure after acid dilution with butanol and triton. Frequent values were established in fifty four deceased biological fluids. As regards the urine samples, the results were corrected after enzymatic creatinine determination. Various types of blood collections were tested. Global performance was evaluated by a specific quality control program. Our laboratory is a registered participant of the National Institute of Public Health of Quebec (Institut National de Santé Publique du Quebec) Sainte-Foy, Canada, inter-laboratory comparison program for whole blood, urine and hair. The limits of detection and the limits of quantitation were considered excellent and the quality control scores good. Usual values for the 54 deceased are presented. Multi-elementary analysis in a single run is most useful for exposure evaluation to poisons (As, $\mathrm{Tl}$ ), heavy metals $(\mathrm{Pb}, \mathrm{Cd}, \mathrm{Hg})$, various metals ( $\mathrm{Al}, \mathrm{Co}$, $\mathrm{Ni}, \mathrm{Mo}, \mathrm{W}$ ), drugs (Li, Pt, Bi, Al), pesticides (As, Pb, Br, Al, $\mathrm{Ba}, \mathrm{Hg}, \mathrm{Se}, \mathrm{Cu}$ ), fumigant bombs ( $\mathrm{Zn}, \mathrm{Al})$ or halogens $(\mathrm{Br}, \mathrm{I})$, lanthanides (Ce, La, Gd) or uranides (Th, $U$ ). The interest of strontium and bromide determinations for death by drowning is mentioned. This multi-elementary procedure is applicable to other matrix like hair or nails. ICP-MS multi-elementary biological matrices should be routinely performed for all unexplained death.

\section{KEY-WORDS}

ICP-MS, forensic, metals, whole blood, urines.

très performantes pour de nombreux xénobiotiques organiques : médicaments, pesticides, substances variées. Cependant, elles ne permettent pas de mettre en évidence des poisons minéraux. Pour ces derniers, un certain nombre d'analyses spécifiques, longues et fastidieuses (cyanures, métaux, métalloïdes) sont nécessaires avec au minimum les dosages des cyanures et du lithium dans le cadre de l'expertise toxicologique 
de référence. Parmi les investigations complémentaires, le dosage des métaux et métalloïdes est le plus souvent effectué par spectrométrie d'absorption atomique en mode monoélémentaire. Aussi nous proposons le dosage simultané d'une trentaine de métaux et métalloïdes dans les prélèvements autopsiques sanguins et urinaires par plasma à couplage inductif relié à un spectromètre de masse (ICP-MS) pour rechercher une exposition à l'un de ces éléments. Récemment, les auteurs ont présenté la validation d'une technique de dosage multiélémentaire des métaux chez l'homme pour 24 éléments dans le sang total, pour 27 éléments dans le plasma et pour 29 éléments dans les urines par ICP-MS (1). Ils ont ensuite déterminé les valeurs usuelles dans ces milieux biologiques chez 100 témoins (2). La mesure des concentrations usuelles dans les cheveux de 45 volontaires non exposés professionnellement a également été réalisée (3). Une synthèse reprend l'ensemble de ces résultats (4). Il nous a semblé utile de compléter ces travaux par la mesure des valeurs fréquentes dans le sang et les urines de sujets décédés. En effet, lors de l'établissement des valeurs usuelles chez 100 témoins, nous avons constaté des concentrations moyennes beaucoup plus faibles que celles généralement admises pour de nombreux éléments (2). Ces valeurs s'expliquent par l'amélioration de la qualité des réactifs et des matériels de prélèvement, mais aussi et surtout grâce aux progrès réalisés dans la spécificité des mesures, en spectrophotométrie d'absorption atomique électrothermique (SAAE) avec la correction du signal non spécifique de type deutérium puis électromagnétique de type Zeeman. L'utilisation de l'ICP-MS, dont les performances sont encore très supérieures à la SAAE tant au plan de la sensibilité que de la spécificité permet ainsi de diminuer les normes établies pour de nombreux éléments. Le second intérêt de l'ICP-MS est la quantification possible d'éléments pour lesquels la mesure par SAAE s'avère impossible ou dont la sensibilité est insuffisante par cette technique. Il est par exemple possible de quantifier des halogénures $(\mathrm{Br}, \mathrm{I})$, des lanthanides (Ce, La, Gd), des actinides $(\mathrm{Th}, \mathrm{U})$. La troisième raison à la réalisation de ces dosages par ICP-MS est la détermination multiélémentaire avec une prise d'essai réduite $(0,4 \mathrm{~mL})$. Compte tenu des nombreuses modifications biologiques qui interviennent immédiatement après la mort, les valeurs normales établies chez des sujets vivants ne sont pas utilisables. Au premier rang des mécanismes responsables figure la lyse cellulaire, phénomène qui s'accompagne de la libération d'éléments intracellulaires dans le torrent circulatoire immédiatement après le décès témoignant de la perte de contrôle de processus actifs comme c'est le cas avec le cadmium par exemple. Il nous a de ce fait paru inutile d'évaluer les concentrations sériques.

\section{Matériel et méthodes}

\section{Instrumentation}

Il s'agit d'une torche à plasma de type ThermoOptek $\mathrm{X}$ Series couplée à un spectromètre de masse, modèle X7/CCT (ThermoOptek, Courtabœuf, France). L'appareil est équipé d'une torche en quartz de 1,5 $\mathrm{mm}$, d'un nébuliseur concentrique en verre borosilicaté de $1 \mathrm{~mL}$ (type Meinhard de Marque Glass Expansion-Référence ThermoElemental 1201318) avec un débit d'échantillon de $0,85 \mathrm{~mL} / \mathrm{min}$, d'une chambre simple de nébulisation à bille d'impact en quartz munie d'un refroidisseur à effet Peltier régulant sa température à $3^{\circ} \mathrm{C}$, et d'un passeur d'échantillons de type CETAC ASX-510. L'ensemble des données est enregistré à l'aide d'une station informatique dotée du logiciel d'analyse Plasmalab ${ }^{\circledR}$ version 2.0 sous Windows NT. Les paramètres instrumentaux sont les suivants : puissance de la torche $1200 \mathrm{~W}$; débits d'argon : plasmagène $15 \mathrm{~L} / \mathrm{min}$, nébuliseur $0,95 \mathrm{~L} / \mathrm{min}$, auxiliaire $0,66 \mathrm{~L} / \mathrm{min}$; interface : cônes échantillonneurs et écorceurs en nickel de diamètre respectifs de $1 \mathrm{~mm}$ et de $0,4 \mathrm{~mm}$; vide au niveau de l'interface : 1,9 mbar et du quadripôle : $1,6.10^{-7}$ mbar. Le dosage de la créatinine urinaire est réalisé par technique enzymatique sur Workcell (Siemens, Puteaux, France).

\section{Matériel de prélèvement}

Pour les sujets vivants, le sang est prélevé dans un tube sous vide de $7 \mathrm{~mL}$ hépariné pour éléments traces de type « Vacutainer ${ }^{\circledR}$ » référence 367735 (BD diagnostics, Le Pont de Claix, France). Ce tube est également utilisé pour le zinc. La moitié de chaque prélèvement sanguin est décantée dans un tube en polystyrène de $5 \mathrm{~mL}$ puis centrifugée dans un délai de 2 heures après le prélèvement et le plasma immédiatement décanté. Le sang total, le plasma décanté sont congelés avant analyse. En ce qui concerne le sang de cadavre, en raison des difficultés à obtenir les prélèvements médico-légaux destinés à ces examens dans des tubes pour éléments traces, deux types de contenants ont été utilisés : des flacons ronds en verre ordinaire $\mathrm{T} 3 \mathrm{de} 15 \mathrm{~mL}$; contenant du fluorure de sodium (nécessaires pour alcoolémie référence BP PREL, TechniDome, Le Cendre, France), des tubes en polyéthylène téréphtalate (PET) de $4 \mathrm{~mL}$ contenant du fluorure de sodium et de l'oxalate de potassium référence 368921 (BD diagnostics, Le Pont de Claix, France). Les urines correspondantes sont recueillies comme pour les sujets vivants dans un flacon de $60 \mathrm{~mL}$ en polystyrène transparent muni d'un bouchon à vis en polypropylène référence TP 35 -007 (Gosselin, Hazbrouck, France). 


\section{Volontaires}

Ce sont 21 sujets volontaires sains chez lesquels un prélèvement sanguin a été réalisé à l'aide des trois contenants décrits précédemment. Le matériel utilisé est une seringue de $20 \mathrm{~mL}$ en polypropylène munie d'un joint en caoutchouc synthétique siliconé référence 003022710 (Pentaferté, Villeparisis, France), et sur laquelle une aiguille $19 \mathrm{G}-1,10 \times 30 \mathrm{~mm}$ en inox est collée avec une colle époxy sur une embase crème en polypropylène, référence 004021B14 (Pentaferté, Villeparisis, France).

\section{Sujets décédés}

Il s'agit de prélèvements réalisés chez 54 sujets des deux sexes dans le cadre de demandes d'expertises toxicologiques aux fins de recherche des causes de la mort pour lesquels du sang fluoré, périphérique le plus souvent, a été recueilli soit dans un flacon fluoré en verre ordinaire, soit dans un tube en PET.

\section{Réactifs}

La source de plasma utilisée est de l'argon présentant un haut degré de pureté, supérieur à 99,999\% (Linde gas, Gargenville, France). L'eau utilisée est purifiée extemporanément sur Milli-QPLUS 185 (Millipore, St Quentin en Yvelines, France). L'acide nitrique à $65 \%$ de qualité suprapur, le 1-butanol, le triton X100 et les solutions multiélémentaires (multiélément standard solution VI contenant 30 éléments à 10 ou $100 \mathrm{mg} / \mathrm{L}$ ) proviennent de chez Merck (Darmstadt, Allemagne). Des étalons individuels de W, Pt, Pd, Hg, Sb, Sn, Ge (CPI international, Amsterdam, Hollande) sont ajoutés à ces solutions multiélémentaires. La détermination du brome est effectuée de manière semi-quantitative. L'étalonnage interne de l'appareil est réalisé avec une solution de Rhodium (CPI international). La validité des dosages est vérifiée pour le sang total et les urines par des contrôles titrés par ICP-MS Seronorm niveau 1 (Trace elements whole blood 2 - réf. 201607 ; Trace elements urine - réf. 201207 ; SERO AS, Billingstade, Norvège). Le laboratoire participe au contrôle qualité ICP-MS canadien (Programme de comparaisons interlaboratoires, Sainte Foy, Canada) qui adresse trois fois par an à ses adhérents du sang total, des urines et des cheveux.

- Préparation des gammes d'étalonnage :

Gammes d'étalonnage permettant le dosage multiélémentaire dans le sang total et les urines :

Dans le sang, le dosage multiélémentaire est effectué par la méthode des ajouts dosés, par une gamme de concentrations dans la matrice considérée, préparée à l'aide d'un pool de sang. Une gamme d'ajouts dosés est réalisée, de X à X+24 $\mu \mathrm{g} / \mathrm{L}(\mathrm{X}, \mathrm{X}+0,5, \mathrm{X}+1, \mathrm{X}+3, \mathrm{X}+6$, $\mathrm{X}+12$ et $\mathrm{X}+24 \mu \mathrm{g} / \mathrm{L})$ ou de $\mathrm{X}$ à $\mathrm{X}+240 \mu \mathrm{g} / \mathrm{L}(\mathrm{X}, \mathrm{X}+5$,
$\mathrm{X}+10, \mathrm{X}+30, \mathrm{X}+60, \mathrm{X}+120$ et $\mathrm{X}+240 \mu \mathrm{g} / \mathrm{L}$ ) selon les éléments. Pour les urines, celles-ci sont diluées au 1/5 avec une gamme selon la méthode des ajouts dosés, à partir d'un pool d'urines, dans les mêmes conditions et aux mêmes concentrations que pour le sang total.

- Préparation des échantillons :

Les échantillons de sang sont dilués au dixième dans une solution aqueuse composée d'acide nitrique $0,144 \mathrm{M}$, de $0,5 \%$ de 1-butanol et de triton $0,1 \%(\mathrm{v} / \mathrm{v})$ dans des tubes en polypropylène. Une fois l'hémolyse achevée, les mélanges sont centrifugés à 4000 tours/min. Les urines sont préparées par dilution au $1: 5$ [acide nitrique $0,216 \mathrm{M}, 0,5 \%$ de 1-butanol, triton $0,01 \%$ $(\mathrm{v} / \mathrm{v})]$. Les résultats sont ensuite rapportés à la créatinine comme pour les sujets vivants (2).

\section{Les outils mathématiques et statistiques}

Pour établir les valeurs usuelles fréquentes dans le sang total et les urines, il est nécessaire d'indiquer une valeur moyenne ainsi qu'un indicateur de dispersion. On utilise communément la moyenne arithmétique et l'écart type. Cependant l'apparition de valeurs s'écartant nettement des autres pèse considérablement sur les calculs. Pour cette raison nous avons utilisé la médiane et les percentiles, indicateurs beaucoup plus robustes aux valeurs extrêmes $(5,6)$.

\section{Résultats}

Il sont exprimés en $\mu \mathrm{g} / \mathrm{L}(=\mathrm{ng} / \mathrm{mL}$ ou ppb) dans le sang (tableau I) et en $\mu \mathrm{g} / \mathrm{g}$ de créatinine dans les urines (tableau II) des cinquante quatre sujets décédés. Les résultats obtenus pour le sang recueilli dans les trois types de contenants (tubes pour éléments traces, flacons fluorés en verre, tubes fluorés en PET) chez les 21 volontaires sont regroupés tableau III. Dans chaque tableau, les valeurs médianes et moyennes, la dispersion des concentrations mesurées, des éléments sont indiquées pour $90 \%$ de la population étudiée du $5^{5^{\text {ème }}}$ au $95^{\text {ème }}$ percentile.

Résultats sanguins :

L'examen du tableau I concernant les résultats obtenus dans le sang des 54 sujets décédés montre des concentrations beaucoup plus importantes que pour les 100 témoins (2) pour les éléments suivants : $\mathrm{B}$ ( 2 à 10 fois), $\mathrm{Al}$ (20 à 25 fois), $\mathrm{Mn}$ (6 à 10 fois), Cd (100 à 130 fois), Sn (10 à 40 fois), W (12 à 50 fois), Bi (7 à 20 fois).

Enrevanche,onnotelephénomèneinverse pour le gallium et le baryum dont les médianes sont respectivement 15 à 50 fois plus faibles que chez les sujets vivants (2). L'évaluation des trois contenants réalisée avec le sang de 21 volontaires (tableau III) confirme ou révèle que les flacons fluorés en verre ordinaire libèrent des quantités importantes d'aluminium et d'étain, les tubes 
Tableau I : Valeurs fréquentes obtenues par ICP-MS dans le sang de 54 sujets décédés. Les résultats sont exprimés en $\mu \mathrm{g} / \mathrm{L} \mathrm{ou} \mathrm{ng} / \mathrm{mL}$.

\begin{tabular}{|c|c|c|c|c|}
\hline Éléments & Moyenne & Médiane & $\begin{array}{c}5^{\text {ème }} \\
\text { percentile }\end{array}$ & $\begin{array}{c}95^{\text {̀̀me }} \\
\text { percentile }\end{array}$ \\
\hline${ }^{7} \mathbf{L i}$ & 5,43 & 0,71 & 0,34 & 17,7 \\
\hline${ }^{9} \mathbf{B e}$ & 0,09 & 0,08 & 0,02 & 0,20 \\
\hline${ }^{11} \mathbf{B}$ & 139 & 57,8 & 27,5 & 452 \\
\hline${ }^{27} \mathbf{A I}$ & 55,0 & 29,2 & 7,48 & 176 \\
\hline${ }^{55} \mathbf{M n}$ & 52,4 & 44,6 & 7,08 & 128 \\
\hline${ }^{59} \mathrm{Co}$ & 1,06 & 0,68 & 0,21 & 3,05 \\
\hline${ }^{60} \mathbf{N i}$ & 4,13 & 3,07 & 1,21 & 11,5 \\
\hline${ }^{65} \mathrm{Cu}$ & 1142 & 1088 & 687 & 1993 \\
\hline${ }^{66} \mathbf{Z n}$ & 9447 & 9437 & 3789 & 16544 \\
\hline${ }^{69} \mathbf{G a}$ & 1,04 & 0,21 & 0,06 & 4,84 \\
\hline${ }^{72} \mathbf{G e}$ & 17,8 & 16,2 & 9,00 & 32,0 \\
\hline${ }^{75} \mathbf{A s}$ & 4,18 & 2,30 & 0,32 & 12,9 \\
\hline${ }^{81} \mathbf{B r}^{*}$ & 2109 & 1768 & 1016 & 4610 \\
\hline${ }^{82} \mathbf{S e}$ & 111 & 105 & 62,6 & 186 \\
\hline${ }^{85} \mathbf{R b}$ & 2977 & 2911 & 1794 & 4558 \\
\hline${ }^{88} \mathbf{S r}$ & 7,39 & 4,06 & 1,03 & 22,7 \\
\hline${ }^{98} \mathbf{M o}$ & 20,2 & 8,71 & 1,00 & 62,8 \\
\hline${ }^{105} \mathbf{P d}$ & 0,10 & 0,04 & $<0,01$ & 0,54 \\
\hline${ }^{107} \mathbf{A g}$ & 0,48 & 0,22 & 0,04 & 1,25 \\
\hline${ }^{111} \mathbf{C d}$ & 65,1 & 33,4 & 1,25 & 261 \\
\hline${ }^{118} \mathbf{S n}$ & 19,8 & 11,0 & 0,38 & 66,5 \\
\hline${ }^{121} \mathbf{S b}$ & 2,70 & 0,14 & 0,03 & 8,10 \\
\hline${ }^{125} \mathrm{Te}$ & 0,27 & 0,24 & 0,14 & 0,53 \\
\hline${ }^{137} \mathbf{B a}$ & 21,5 & 1,07 & 0,18 & 111 \\
\hline${ }^{182} \mathbf{W}$ & 0,13 & 0,08 & 0,03 & 0,46 \\
\hline${ }^{195} \mathbf{P t}$ & 0,01 & $<0,01$ & $<0,01$ & 0,04 \\
\hline${ }^{202} \mathrm{Hg}$ & 4,77 & 3,57 & 0,63 & 13,9 \\
\hline${ }^{205} \mathbf{T I}$ & 0,11 & 0,09 & 0,03 & 0,22 \\
\hline${ }^{208} \mathbf{P b}$ & 65,0 & 49,1 & 9,47 & 184 \\
\hline${ }^{209} \mathbf{B} \mathbf{i}$ & 0,04 & $<0,01$ & $<0,01$ & 0,17 \\
\hline${ }^{238} \mathbf{U}$ & 0,04 & 0,01 & $<0,01$ & 0,13 \\
\hline
\end{tabular}

Tableau II : Valeurs fréquentes obtenues par ICP-MS dans les urines de 54 sujets décédés. Les résultats sont exprimés en $\mu \mathrm{g} / \mathrm{g}$ de créatinine.

\begin{tabular}{|c|c|c|c|c|}
\hline Éléments & Moyenne & Médiane & $\begin{array}{c}5^{\text {ème }} \\
\text { percentile }\end{array}$ & $\begin{array}{c}95^{\text {ème }} \\
\text { percentile }\end{array}$ \\
\hline${ }^{7} \mathbf{L i}$ & 22,1 & 13,3 & 2,04 & 62,8 \\
\hline${ }^{9} \mathbf{B e}$ & 0,10 & 0,06 & $<0,01$ & 0,25 \\
\hline${ }^{11} \mathbf{B}$ & 1323 & 651 & 116 & 3156 \\
\hline${ }^{27} \mathbf{A I}$ & 8,02 & 4,03 & 0,55 & 13,9 \\
\hline${ }^{51} \mathbf{V}$ & 2,69 & 2,19 & 0,39 & 7,89 \\
\hline${ }^{55} \mathbf{M n}$ & 8,00 & 2,53 & 0,50 & 21,1 \\
\hline${ }^{59} \mathrm{Co}$ & 0,67 & 0,44 & 0,17 & 1,18 \\
\hline${ }^{60} \mathbf{N i}$ & 6,01 & 3,47 & 0,31 & 11,3 \\
\hline${ }^{65} \mathrm{Cu}$ & 80,1 & 34,2 & 8,96 & 348 \\
\hline${ }^{66} \mathbf{Z n}$ & 3974 & 1415 & 359 & 5329 \\
\hline${ }^{69} \mathbf{G a}$ & 0,16 & 0,12 & 0,03 & 0,42 \\
\hline${ }^{72} \mathbf{G e}$ & 1,08 & 0,76 & 0,02 & 3,20 \\
\hline${ }^{75} \mathbf{A S}$ & 67,4 & 10,0 & 2,63 & 293 \\
\hline${ }^{81} \mathbf{B r}^{*}$ & 2871 & 1932 & 648 & 6343 \\
\hline${ }^{82} \mathbf{S e}$ & 48,1 & 31,3 & 17,6 & 102 \\
\hline${ }^{85} \mathbf{R b}$ & 2656 & 2133 & 473 & 5013 \\
\hline${ }^{88} \mathbf{S r}$ & 169 & 114 & 18,0 & 494 \\
\hline${ }^{98} \mathbf{M o}$ & 19,3 & 14,2 & 2,65 & 40,4 \\
\hline${ }^{105} \mathbf{P d}$ & 0,32 & 0,21 & 0,06 & 0,70 \\
\hline${ }^{107} \mathbf{A g}$ & 0,16 & 0,08 & 0,01 & 0,46 \\
\hline${ }^{111} \mathbf{C d}$ & 4,38 & 1,01 & 0,12 & 17,8 \\
\hline${ }^{118} \mathbf{S n}$ & 1,21 & 0,45 & 0,08 & 4,31 \\
\hline${ }^{121} \mathbf{S b}$ & 0,17 & 0,11 & 0,03 & 0,61 \\
\hline${ }^{125} \mathrm{Te}$ & 0,47 & 0,34 & 0,10 & 1,50 \\
\hline${ }^{137} \mathbf{B a}$ & 2,42 & 1,76 & 0,32 & 6,84 \\
\hline${ }^{182} \mathbf{W}$ & 0,15 & 0,08 & 0,02 & 0,50 \\
\hline${ }^{195} \mathbf{P t}$ & 0,01 & $<0,01$ & $<0,01$ & 0,04 \\
\hline${ }^{202} \mathbf{H g}$ & 2,65 & 1,72 & 0,29 & 8,93 \\
\hline${ }^{205} \mathbf{T I}$ & 0,27 & 0,14 & 0,05 & 0,76 \\
\hline${ }^{208} \mathbf{P b}$ & 2,26 & 1,47 & 0,16 & 6,52 \\
\hline${ }^{209} \mathbf{B i}$ & 0,07 & 0,02 & $<0,01$ & 0,26 \\
\hline${ }^{238} \mathbf{U}$ & 0,01 & $<0,01$ & $<0,01$ & 0,04 \\
\hline
\end{tabular}

Tableau III : Concentration de 12 éléments dans le sang de 21 témoins prélevé avec 3 contenants différents.

\begin{tabular}{|c|c|c|c|c|c|}
\hline Éléments & Contenant & Moyenne & Médiane & $\begin{array}{c}5^{\text {ème }} \\
\text { percentile }\end{array}$ & $\begin{array}{c}95^{\text {ème }} \\
\text { percentile }\end{array}$ \\
\hline \multirow{3}{*}{${ }^{11} \mathbf{B}$} & ET & 49,7 & 49,5 & 30,1 & 80,3 \\
\hline & PET & 1356 & 1467 & 536 & 2123 \\
\hline & FV & 79,3 & 77,2 & 50,3 & 126 \\
\hline \multirow{3}{*}{${ }^{27} \mathbf{A I}$} & ET & 4,78 & 4,80 & 2,84 & 6,64 \\
\hline & PET & 11,8 & 10,2 & 8,40 & 19,3 \\
\hline & $\mathrm{FV}$ & 52,9 & 46,5 & 36,8 & 80,5 \\
\hline \multirow{3}{*}{${ }^{55} \mathbf{M n}$} & ET & 9,50 & 9,15 & 6,34 & 13,0 \\
\hline & PET & 9,88 & 10,2 & 6,50 & 13,6 \\
\hline & FV & 8,83 & 8,48 & 5,94 & 16,5 \\
\hline \multirow{3}{*}{${ }^{60} \mathbf{N i}$} & ET & 2,81 & 2,86 & 1,58 & 4,39 \\
\hline & PET & 7,21 & 7,69 & 3,88 & 11,5 \\
\hline & FV & 2,92 & 2,87 & 1,45 & 5,13 \\
\hline \multirow{3}{*}{${ }^{69} \mathbf{G a}$} & ET & 2,59 & 2,35 & 2,15 & 3,63 \\
\hline & PET & 0,34 & 0,37 & 0,09 & 0,56 \\
\hline & FV & 0,13 & 0,11 & 0,04 & 0,29 \\
\hline \multirow{3}{*}{${ }^{75} \mathbf{A s}$} & ET & 2,83 & 2,45 & 1,60 & 6,3 \\
\hline & PET & 2,60 & 2,20 & 1,38 & 6,20 \\
\hline & FV & 2,60 & 2,26 & 1,39 & 5,40 \\
\hline \multirow{3}{*}{${ }^{85} \mathbf{R b}$} & ET & 1866 & 1834 & 1549 & 2254 \\
\hline & PET & 1760 & 1780 & 1328 & 2156 \\
\hline & FV & 1706 & 1669 & 1284 & 2250 \\
\hline \multirow{3}{*}{${ }^{88} \mathbf{S r}$} & ET & 20,1 & 18,9 & 13,2 & 33,5 \\
\hline & PET & 18,1 & 18,3 & 8,22 & 29,2 \\
\hline & FV & 9,65 & 8,41 & 4,16 & 29,6 \\
\hline \multirow{3}{*}{${ }^{111} \mathbf{C d}$} & ET & 0,42 & 0,37 & 0,26 & 1,00 \\
\hline & PET & 0,33 & 0,30 & 0,20 & 0,54 \\
\hline & FV & 0,37 & 0,35 & 0,22 & 0,73 \\
\hline \multirow{3}{*}{${ }^{118} \mathbf{S n}$} & ET & 0,30 & 0,27 & 0,08 & 0,67 \\
\hline & PET & 1,20 & 0,97 & 0,74 & 2,32 \\
\hline & FV & 26,0 & 20,7 & 14,1 & 78,0 \\
\hline \multirow{3}{*}{${ }^{121} \mathbf{S b}$} & ET & 0,04 & 0,04 & 0,03 & 0,06 \\
\hline & PET & 2,80 & 2,81 & 1,63 & 4,06 \\
\hline & FV & 0,09 & 0,06 & 0,04 & 0,41 \\
\hline \multirow{3}{*}{${ }^{137} \mathbf{B a}$} & ET & 46,1 & 44,1 & 40,7 & 59,6 \\
\hline & PET & 5,34 & 6,01 & 1,32 & 9,79 \\
\hline & FV & 1,45 & 1,02 & 0,11 & 4,72 \\
\hline
\end{tabular}

fluorés en PET augmentent artificiellement le bore, l'antimoine, le nickel, alors que les tubes héparinés en verre pour éléments traces contiennent des quantités non négligeables de baryum et de gallium.

Résultats urinaires :

L'analyse du tableau II regroupant l'ensemble des dosages urinaires des 54 sujets décédés montre des concentrations beaucoup plus importantes que pour les 100 témoins (2) pour les éléments suivants : $\mathrm{Be}$ (6 fois), $\mathrm{Mn}$ (8 à 16 fois), $\mathrm{Cu}$ (5 à 29 fois), $\mathrm{Zn}$ (7 à 11 fois), $\mathrm{Cd}$ (6 à 22 fois), Bi (21 à 29 fois).

\section{Discussion}

L'analyse des résultats obtenus avec les tubes spéciaux pour éléments traces chez les 21 sujets volontaires montre des augmentations très nettes pour le gallium et le baryum faussant ainsi les valeurs «normales » établies avec ce type de contenant pour les deux éléments. Celles-ci sont liées à la présence de ${ }^{69} \mathrm{Ga}$ dans les tubes qui avec son doublement chargé de masse 138 majore et fausse le 
dosage de ${ }^{137} \mathrm{Ba}$ en raison d'une interférence isotopique. Contrairement au sujet vivant pour lequel il est relativement aisé de disposer de prélèvements sanguins réalisés sur des tubes pour éléments traces, cela est plus difficile à organiser sur le cadavre en raison de l'origine géographique variée de ces prélèvements et de la multitude d'interlocuteurs. En effet, un certain nombre de prélèvements sont réalisés en dehors du circuit de la médecine légale : médecins d'urgence (SAMU, SMUR), médecins libéraux. Par ailleurs, seul un nombre limité de cas justifie la réalisation d'un bilan par ICP-MS. Or la qualité du prélèvement pour ce type d'analyse est tout à fait primordiale afin d'éviter toute mauvaise interprétation liée à une pollution éventuelle. C'est la raison pour laquelle chez les sujets autopsiés, nous préconisons l'ajout systématique dans le kit de prélèvement d'un tube spécial pour le sang (7). L'examen des résultats montre à l'évidence qu'à l'heure actuelle aucun contenant n'est satisfaisant pour le sang, qu'il s'agisse d'un sujet vivant ou d'un sujet décédé contrairement aux urines où les flacons en polystyrène avec bouchons en polypropylène n'apportent aucune contamination notable. La quantification dans un cadre médico-légal s'intéressant à des concentrations tout à fait considérables par rapport aux concentrations fréquentes, seules des variations de concentration d'un facteur cinq sont discutées.

Dosages sanguins :

Deux types de contenants sont à proscrire : ceux renfermant de l'héparinate de lithium en raison de la présence de lithium et ceux avec merthiolate qui contiennent du mercure (flacons réglementaires pour alcoolémie médico-légale post-mortem). Les résultats post-mortem comparés à ceux obtenus chez les sujets vivants montrent des différences importantes ou très importantes pour quelques éléments. Il s'agit essentiellement d'augmentations. Parmi celles-ci, il convient de différencier celles liées au contenant et celles en rapport avec la libération post-mortem dans le torrent circulatoire. Le dosage chez les 54 sujets décédés a été réalisé le plus souvent dans le sang recueilli sur des flacons en verre ordinaire, ce qui explique des concentrations beaucoup plus élevées en aluminium et en étain, mais également en bore, contaminant des tubes en PET qui ont été utilisés dans un certain nombre de cas. Pour d'autres éléments, la libération post-mortem à partir du contenu cellulaire qui conduit à des augmentations très importantes pour le manganèse et tout à fait considérables pour le cadmium ont manifestement pour origine la libération des contenus cellulaires. Cependant, même dans ces cas, les concentrations susceptibles d'être mesurées sont au maximum de l'ordre du demi milligramme par litre et n'ont aucune commune mesure avec celles rencontrées dans des intoxications graves ou mortelles impliquant ces éléments (tableau V).

Dosages urinaires :

En l'absence de contamination liée au contenant, identique à celui utilisé chez les témoins vivants, les augmentations constatées sont vraisemblablement liées à la mort des sujets. Il s'agit probablement de la perte du mécanisme actif de concentration cellulaire qui concourt en phase pré-agonique ou agonique à une élimination urinaire accrue. Ce phénomène pourrait être majoré par l'atteinte rénale. Il concerne deux éléments pour lesquels les concentrations cellulaires sont importantes : le cadmium et le manganèse.

Interprétation globale :

La réalisation systématique de bilans sanguins et urinaires par ICP-MS nous semble utile. Elle permet de s'assurer qu'en principe aucune contamination n'est intervenue avec les contenants grâce à l'analyse des deux milieux. Ainsi, les élévations du bore et de l'aluminium dans le sang de cadavre semblent bien liées à l'emploi du verre pour le recueil (Tableau VII) car les concentrations urinaires des sujets décédés sont voisines de celles des témoins vivants. Ceci est confirmé par l'analyse du sang des 21 volontaires dans différents contenants

Tableau $\boldsymbol{V}$ : Comparaison des concentrations sanguines chez les sujets vivants et chez les sujets décédés (5ème-95ème percentile).

\begin{tabular}{|c|c|c|}
\hline Éléments & $\begin{array}{c}\text { Sujets vivants } \\
(n=100)\end{array}$ & $\begin{array}{c}\text { Sujets décédés } \\
(n=54)\end{array}$ \\
\hline${ }^{7} \mathbf{L i}$ & $<33$ & $0,34-17,7$ \\
\hline${ }^{9} \mathbf{B e}$ & $0,02-0,09$ & $0,02-0,20$ \\
\hline${ }^{11} \mathbf{B}$ & $14-44$ & $27,0-452$ \\
\hline${ }^{27} \mathbf{A I}$ & $1,28-6,35$ & $7,48-176$ \\
\hline${ }^{55} \mathrm{Mn}$ & $5,0-12,8$ & $7,1-128$ \\
\hline${ }^{59} \mathrm{Co}$ & $0,04-0,64$ & $0,21-3,05$ \\
\hline${ }^{60} \mathbf{N i}$ & $0,09-4,18$ & $1,21-11,5$ \\
\hline${ }^{65} \mathrm{Cu}$ & $600-1500$ & $687-1993$ \\
\hline${ }^{66} \mathbf{Z n}$ & $4000-7500$ & $3789-16544$ \\
\hline${ }^{69} \mathbf{G a}$ & $2,65-4,71$ & $0,06-4,84$ \\
\hline${ }^{72} \mathbf{G e}$ & $10,8-19,5$ & $9,00-32,0$ \\
\hline${ }^{75} \mathrm{As}$ & $2,6-17,8$ & $0,32-18,5$ \\
\hline${ }^{81} \mathbf{B r} *$ & - & $1016-4610$ \\
\hline${ }^{82} \mathbf{S e}$ & $89-154$ & $63-186$ \\
\hline${ }^{85} \mathbf{R b}$ & $1289-2358$ & $1794-4558$ \\
\hline${ }^{88} \mathbf{S r}$ & $9-41$ & $1,00-23,0$ \\
\hline${ }^{98} \mathbf{M o}$ & $0,77-7,86$ & $1,00-63,0$ \\
\hline${ }^{105} \mathbf{P d}$ & $0,01-0,71$ & $<0,01-0,54$ \\
\hline${ }^{107} \mathrm{Ag}$ & $0,69-4,51$ & $0,04-1,25$ \\
\hline${ }^{111} \mathbf{C d}$ & $0,15-2,04$ & $1,25-261$ \\
\hline${ }^{118} \mathbf{S n}$ & $0,11-1,75$ & $0,38-66,0$ \\
\hline${ }^{121} \mathbf{S b}$ & $0,05-0,13$ & $0,03-8,10$ \\
\hline${ }^{125} \mathbf{T e}$ & $0,11-0,45$ & $0,14-0,53$ \\
\hline${ }^{137} \mathbf{B a}$ & $46,4-77,6$ & $0,18-111$ \\
\hline${ }^{182} \mathbf{W}$ & $<0,01-0,08$ & $0,03-0,46$ \\
\hline${ }^{195} \mathbf{P t}$ & $<0,01-0,01$ & $<0,01-0,04$ \\
\hline${ }^{202} \mathrm{Hg}$ & $0,94-8,13$ & $0,63-13,9$ \\
\hline${ }^{205} \mathbf{T l}$ & $0,01-0,04$ & $0,03-0,22$ \\
\hline${ }^{208} \mathbf{P b}$ & $11,4-62,8$ & $9,5-184$ \\
\hline${ }^{209} \mathbf{B i}$ & $<0,01-<0,01$ & $<0,01-0,17$ \\
\hline${ }^{238} \mathbf{U}$ & $<0,01-<0,01$ & $<0,01-0,13$ \\
\hline
\end{tabular}


Tableau IV : Évaluation des différents contenants.

\begin{tabular}{|c|c|c|c|c|}
\hline Contenant & Matériau & Additif(s) & $\begin{array}{c}\text { Usage } \\
\text { principal }\end{array}$ & $\begin{array}{c}\text { Éléments } \\
\text { augmentés }\end{array}$ \\
\hline $\begin{array}{c}\text { Tube de } \\
\mathbf{7 m L} \text { pour } \\
\text { éléments } \\
\text { traces }\end{array}$ & Verre spécial & $\begin{array}{c}\text { Héparinate de } \\
\text { sodium }\end{array}$ & $\begin{array}{c}\text { Sujets vivants : } \\
\text { Éléments } \\
\text { traces }\end{array}$ & $\begin{array}{c}\text { Baryum } \\
\text { Gallium }\end{array}$ \\
\hline $\begin{array}{c}\text { Flacon de } \\
\mathbf{1 5} \mathbf{~ m L}\end{array}$ & $\begin{array}{c}\text { Verre } \\
\text { ordinaire }\end{array}$ & $\begin{array}{c}\text { Fluorure de } \\
\text { sodium }\end{array}$ & $\begin{array}{c}\text { Post-mortem: } \\
\text { Alcoolémie } \\
\text { Toxicologie }\end{array}$ & $\begin{array}{c}\text { Aluminium } \\
\text { Étain }\end{array}$ \\
\hline $\begin{array}{c}\text { Tube de } \\
\mathbf{5} \mathbf{~ m L}\end{array}$ & PET & $\begin{array}{c}\text { Fluorure } \\
\text { de sodium } \\
\text { Oxalate de } \\
\text { potassium }\end{array}$ & $\begin{array}{c}\text { Post-mortem: } \\
\text { Alcoolémie } \\
\text { Toxicologie }\end{array}$ & $\begin{array}{c}\text { Antimoine } \\
\text { Bore } \\
\text { Nickel }\end{array}$ \\
\hline
\end{tabular}

Tableau VI : Comparaison des concentrations urinaires chez les

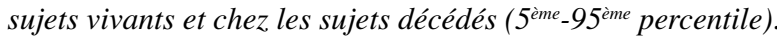

\begin{tabular}{|c|c|c|}
\hline Éléments & $\begin{array}{c}\text { Sujets vivants } \\
(\mathbf{n = 1 0 0})\end{array}$ & $\begin{array}{c}\text { Sujets décédés } \\
(\mathbf{n}=\mathbf{5 4})\end{array}$ \\
\hline${ }^{7} \mathbf{L i}$ & $4,60-219$ & $2,00-63,0$ \\
\hline${ }^{9} \mathbf{B e}$ & $<0,01-0,04$ & $<0,01-0,25$ \\
\hline${ }^{11} \mathbf{B}$ & $282-2072$ & $116-3156$ \\
\hline${ }^{27} \mathbf{A I}$ & $0,6-11,2$ & $0,55-13,9$ \\
\hline${ }^{51} \mathbf{V}$ & $1,4-10,2$ & $0,39-7,9$ \\
\hline${ }^{55} \mathbf{M n}$ & $0,11-1,32$ & $0,50-21,0$ \\
\hline${ }^{59} \mathbf{C o}$ & $0,16-1,14$ & $0,17-1,18$ \\
\hline${ }^{60} \mathbf{N i}$ & $0,59-4,06$ & $0,31-11,3$ \\
\hline${ }^{65} \mathbf{C u}$ & $4,30-12,1$ & $9,00-348$ \\
\hline${ }^{66} \mathbf{Z n}$ & $44,0-499$ & $359-5329$ \\
\hline${ }^{69} \mathbf{G a}$ & $0,02-0,28$ & $0,03-0,42$ \\
\hline${ }^{72} \mathbf{G e}$ & $1,17-3,37$ & $0,02-3,20$ \\
\hline${ }^{75} \mathbf{A s}$ & $2,30-161$ & $2,60-293$ \\
\hline${ }^{81} \mathbf{B r} *$ & & $0,00-4856$ \\
\hline${ }^{82} \mathbf{S e}$ & $10,5-45,5$ & $17,60-102$ \\
\hline${ }^{85} \mathbf{R b}$ & $433-2698$ & $473-5013$ \\
\hline${ }^{88} \mathbf{S r}$ & $20,0-413$ & $18,0-491$ \\
\hline${ }^{98} \mathbf{M o}$ & $7,00-50,0$ & $2,60-40,0$ \\
\hline${ }^{105} \mathbf{P d}$ & $0,07-0,64$ & $0,06-0,70$ \\
\hline${ }^{107} \mathbf{A g}$ & & $0,01-0,46$ \\
\hline${ }^{111} \mathbf{C d}$ & $0,06-0,79$ & $0,12-17,8$ \\
\hline${ }^{118} \mathbf{S n}$ & $0,05-2,28$ & $0,08-4,31$ \\
\hline${ }^{121} \mathbf{S b}$ & $0,02-0,08$ & $0,03-0,61$ \\
\hline${ }^{125} \mathbf{T e}$ & $0,10-0,52$ & $0,10-1,50$ \\
\hline${ }^{137} \mathbf{B a}$ & $0,17-3,85$ & $0,32-6,84$ \\
\hline${ }^{182} \mathbf{W}$ & $0,01-0,09$ & $0,02-0,50$ \\
\hline${ }^{195} \mathbf{P t}$ & $<0,01-0,04$ & $<0,01-0,04$ \\
\hline${ }^{202} \mathbf{H g}$ & $0,14-2,21$ & $0,29-8,93$ \\
\hline${ }^{205} \mathbf{T I}$ & $0,07-0,84$ & $0,05-0,76$ \\
\hline${ }^{208} \mathbf{P b}$ & $0,01-2,14$ & $0,16-6,52$ \\
\hline${ }^{209} \mathbf{B i}$ & $<0,01-0,01$ & $<0,01-0,26$ \\
\hline${ }^{238} \mathbf{U}$ & $20,01-0,01$ & $<0,01-0,04$ \\
\hline Les résulats sont exprimés en $\mu \mathrm{g} / \mathrm{L} *$ dosage semi-quantitatif \\
\hline & & \\
${ }^{15}$ & & \\
\hline
\end{tabular}

(Tableau IV). De la même manière, l'augmentation de l'étain sanguin post-mortem est essentiellement liée à l'emploi de tubes en PET (Tableaux IV et VII). Cependant, malgré l'augmentation parfois importante dans le sang de certains métaux ( $\mathrm{B}, \mathrm{Al}, \mathrm{Mn}, \mathrm{Cd}$, Sn) liées soit au contenant, soit à la libération postmortem, les concentrations fréquentes mesurées chez les sujets décédés demeurent très inférieures à celles constatées dans des intoxications graves ou à
Tableau VII : Comparaison des concentrations sanguines et urinaires chez 100 témoins vivants et 54 sujets décédés.

\begin{tabular}{|c|c|c|c|c|}
\hline & \multicolumn{2}{|c|}{$\begin{array}{c}\text { Sang }(\mu \mathrm{g} / \mathrm{L}) \\
\mathbf{5}^{\text {eme }}-\mathbf{9 5}^{\text {eme }} \text { percentile }\end{array}$} & \multicolumn{2}{c|}{$\begin{array}{c}\text { Urines }(\mu \mathrm{g} / \mathrm{g} \text { de créatinine }) \\
\mathbf{5}^{\text {Ème }}-\mathbf{9 5}^{\text {eme }} \text { percentile }\end{array}$} \\
\hline Élément & Vivants & Décédés & Vivants & Décédés \\
\hline B & $14,0-44,0$ & $27-452$ & $282-2072$ & $116-3156$ \\
\hline AI & $1,30-6,30$ & $7,5-176$ & $0,16-11,0$ & $0,55-14,0$ \\
\hline Mn & $5,00-13,0$ & $7,10-128$ & $0,11-1,30$ & $0,50-21,0$ \\
\hline Cd & $0,15-2,00$ & $1,20-261$ & $0,06-0,79$ & $0,12-18,0$ \\
\hline Sn & $0,11-1,70$ & $0,40-66,0$ & $0,05-2,30$ & $0,08-4,30$ \\
\hline
\end{tabular}

conséquence mortelle avec ces éléments. Ainsi, pour le bore, les concentrations toxiques sanguines sont de plusieurs dizaines de milligrammes par litre (8). Pour l'aluminium, les signes d'encéphalopathie n'apparaissent que pour des aluminémies supérieures à $0,2 \mathrm{mg} / \mathrm{L}$ (9) et les décès pour des concentrations voisines du milligramme (10-13). En ce qui concerne le manganèse et le cadmium, l'intoxication par le métal s'accompagne de concentrations supérieures à $0,1 \mathrm{mg} / \mathrm{L}(9)$. Il nous semble cependant souhaitable que chaque laboratoire établisse ses valeurs fréquentes en fonction : des modalités de recueil, du matériel et de la localisation du prélèvement, ainsi que de la nature du contenant. Par ailleurs, seule une élévation significative dans le sang et les urines doit être prise en compte. Quelques exemples issus de l'expérience des auteurs sont présentés :

Observation $\mathrm{n}^{\circ} 1$ : Il s'agit d'une jeune femme dépressive, traitée par Athymil ${ }^{\circledR}$, Nozinan ${ }^{\circledR}$ et $\operatorname{Prozac} \AA$ chez laquelle les concentrations sanguines mesurées des principes actifs et métabolites n'expliquent pas le décès. Le dosage multiélémentaire sanguin montre une concentration potentiellement mortelle de lithium pour un sujet naï $(2,6 \mathrm{mmol} / \mathrm{L})$.

Observation $n^{\circ} 2:$ Un homme de 66 ans après plusieurs jours d'hospitalisation meurt dans un tableau qui évoque une intoxication alimentaire. Les analyses toxicologiques sont négatives. Le dosage multiélémentaire sanguin post-mortem révèle une concentration en arsenic de $713 \mu \mathrm{g} / \mathrm{L}$ (concentrations normales $<13 \mu \mathrm{g} / \mathrm{L}$ ) probablement liée à la consommation d'un aliment empoisonné.

Observation $\mathrm{n}^{\circ} 3$ : Une femme dépressive de 43 ans meurt à son domicile. L'autopsie met en évidence une congestion multiviscérale. L'expertise toxicologique est en faveur d'une ingestion massive de bromazépam et de citalopram. Les concentrations sanguines confirment cette hypothèse et confortent les constatations autopsiques : bromazépam $=1365 \mu \mathrm{g} / \mathrm{L}$ (concentrations thérapeutiques $<170 \mu \mathrm{g} / \mathrm{L})$, citalopram $=2007 \mu \mathrm{g} / \mathrm{L}$ (concentrations thérapeutiques $<200 \mu \mathrm{g} / \mathrm{L}$ ). Le dosage multiélémentaire sanguin montre une bromémie très élevée $(13000 \mu \mathrm{g} / \mathrm{L}$, normales $<4610 \mu \mathrm{g} / \mathrm{L})$ qui permet 
de préciser la forme galénique ingérée : du Séropram $20 ®$ (le Séropam 20® est un bromhydrate, alors que le Séropam $40 \circledR$ est un chlorure).

La technique est également applicable à d'autres matrices biologiques : cheveux et ongles, voire tissus. Les concentrations fréquentes établies dans les cheveux chez 45 témoins vivants sont utilisables chez le cadavre, sous réserve de l'absence de pollution après le décès (Tableau VIII).

Tableau VIII : Valeurs de référence dans les cheveux de 45 témoins des deux sexes.

\begin{tabular}{|c|c|c|c|}
\hline \multirow{2}{*}{$\begin{array}{c}\text { Éléments } \\
\text { Lithium } \\
\end{array}$} & \multirow{2}{*}{$\begin{array}{c}\text { Médiane } \\
0,016 \\
\end{array}$} & \multicolumn{2}{|c|}{$\begin{array}{c}\text { Dispersion } \\
5^{\text {ème }}-95^{\text {ème }} \text { percentile } \\
\text { ng/mg ou } \mu \mathrm{g} / \mathrm{g}\end{array}$} \\
\hline & & 0,003 & - 0,042 \\
\hline Bérylium & 0,007 & 0,003 & $-\quad 0,012$ \\
\hline Bore & 0,54 & 0,26 & $-\quad 1,87$ \\
\hline Aluminium & 1,63 & 0,26 & $\begin{array}{l}, 30 \\
\end{array}$ \\
\hline Vanadium & 0,016 & 0,001 & $-0,051$ \\
\hline Chrome & 0,20 & 0,11 & $-0,52$ \\
\hline Manganèse & 0,067 & 0,016 & - 0,570 \\
\hline Cobalt & 0,023 & 0,004 & $-\quad 0,14$ \\
\hline Nickel & 0,23 & 0,08 & $-0,90$ \\
\hline Cuivre & 20,3 & 9,0 & - 61,3 \\
\hline Zinc & 162 & 129 & $\begin{array}{l}-209 \\
\end{array}$ \\
\hline Gallium & 0,011 & 0,002 & $-0,068$ \\
\hline Germanium & 0,004 & $<0,001$ & - 0,039 \\
\hline Arsenic & 0,05 & 0,03 & $\begin{array}{l}-0,08 \\
\end{array}$ \\
\hline Sélénium & 0,54 & 0,37 & $\begin{array}{l} \\
-\end{array}$ \\
\hline Rubidium & 0,006 & 0,003 & - 0,03 \\
\hline Strontium & 0,89 & 0,17 & $-4,63$ \\
\hline Molybdène & 0,021 & 0,010 & - 0,028 \\
\hline Palladium & 0,010 & 0,004 & $-\quad 0,049$ \\
\hline Argent & 0,08 & 0,02 & - 1,31 \\
\hline Cadmium & 0,011 & 0,004 & $-0,17$ \\
\hline Etain & 0,046 & 0,007 & $\begin{array}{l} \\
-\quad 0,34 \\
\end{array}$ \\
\hline Antimoine & 0,008 & 0,003 & - 0,13 \\
\hline Tellure & 0,0003 & 0,0003 & $\begin{array}{l}-0,001 \\
\end{array}$ \\
\hline Baryum & 0,28 & 0,05 & $\begin{array}{l}-1,58 \\
\end{array}$ \\
\hline Tungstène & 0,0013 & 0,0001 & $-\quad 0,007$ \\
\hline Platine & 0,00035 & 0,00035 & $-\quad 0,0008$ \\
\hline Mercure & 0,66 & 0,31 & $\begin{array}{l}-1,66 \\
\end{array}$ \\
\hline Thallium & 0,0002 & 0,0001 & $-\quad 0,0004$ \\
\hline Plomb & 0,41 & 0,13 & $\begin{array}{l}-4,57 \\
\end{array}$ \\
\hline Bismuth & 0,009 & 0,0004 & $-0,14$ \\
\hline Uranium & 0,009 & 0,002 & $\begin{array}{l}-0,03 \\
\end{array}$ \\
\hline
\end{tabular}

L'ICP-MS constitue un outil puissant pour mettre en évidence une intoxication ou une exposition à divers éléments en médecine légale. Les principales applications sont reportées tableau IX. L'énumération n'est pas exhaustive et le champ d'investigation peut dépasser les éléments listés dans cette présentation. C'est ainsi que nous venons de mettre en évidence une intoxication au gadolinium, lanthanide utilisé en exploration radiologique pour réaliser les examens d'imagerie par
Tableau IX : Principales applications de l'ICP-MS en médecine légale.

\begin{tabular}{|l|}
\hline Reherche d'exposition (sang, urines) \\
\hline Poisons « classiques » : $\mathrm{As}, \mathrm{Tl}$ \\
\hline Métaux lourds : $\mathrm{Pb}, \mathrm{Cd}, \mathrm{Hg}$ \\
\hline Métaux variés : $\mathrm{Al}, \mathrm{Co}, \mathrm{Ni}, \mathrm{Mo}, \mathrm{W}$ \\
\hline Médicaments : $\mathrm{Li}, \mathrm{Br}, \mathrm{Pt}, \mathrm{Al}, \mathrm{Bi}$ \\
\hline Pesticides : As, $\mathrm{Pb}, \mathrm{Al}, \mathrm{Ba}, \mathrm{Hg}, \mathrm{Se}, \mathrm{Cu}, \mathrm{Br}$ \\
\hline Bombes fumigènes militaires : $\mathrm{Zn}, \mathrm{Al}$ \\
\hline Halogénures : $\mathrm{Br}, \mathrm{I}$ \\
\hline Diagnostic de noyade $: \mathrm{Sr}, \mathrm{Br}$ \\
\hline Lanthanides : $\mathrm{Ce}, \mathrm{La}, \mathrm{Gd}$ \\
\hline Uranides : $\mathrm{Th}, \mathrm{U}$ \\
\hline
\end{tabular}

résonance magnétique. Le dosage mutiélémentaire des métaux et métalloïdes devrait être réalisé dans tous les cas de décès inexpliqués par l'autopsie et pour lesquels l'expertise toxicologique de référence s'avère négative.

\section{Remerciements}

Cet équipement a été acquis grâce à un financement de l'Association pour la Fondation Charles Nicolle - Rouen - France (Président Professeur Eric MALLET).

\section{Références}

1. Goullé J.P, Mahieu L., Castermant J., Neveu N., Lainé G., Nouveau M. P., Gehanne R., Bouige D., Lacroix C. Validation d'une technique de dosage multiélémentaire des métaux par ICP-MS dans les milieux biologiques. Ann. Toxicol. Anal. 2003 ; 15 : 271-80. Correctif dans Ann. Toxicol. Anal. $2004 ; 16$ : 257-60.

2. Goullé J.P., Mahieu L., Castermant J., Neveu N., Lainé G., Nouveau M.P., Gehanne R., Bouige D., Lacroix C. Dosage multiélémentaire des métaux et métalloïdes par ICP-MS : valeurs usuelles chez 100 témoins. Ann. Toxicol. Anal. $2004 ; 16: 261-268$.

3. Goullé J.P., Mahieu L., Bonneau L., Lainé G., Bouige D., Lacroix C. Validation d'une technique de dosage multiélémentaire des métaux et métalloïdes dans les cheveux par ICP-MS. Valeurs de référence chez 45 témoins. Ann. Toxicol. Anal. 2005 ; 17: 97-103.

4. Goullé J.P., Mahieu L., Castermant J., Neveu N., Bonneau L., Lainé G., Bouige D., Lacroix C. Metal and metalloid multi-elementary ICP-MS validation in whole blood, plasma, urine and hair. Reference values. Forensic Sci. Int. $2005 ; 153: 39-44$.

5. Lebart L., Morineau A., Piron M. Statistique exploratoire multidimensionnelle. Paris : Dunod, 1995.

6. Apostoli P., Cortesi I., Mangili A. et coll. Assessment of reference values for mercury in urine : the results of an italian polycentric study. Sci. Total Environ. 2002 ; 289 : 13-24. 
7. Pépin G., Deveaux M., Goullé J.P., Kintz P., Marquet P. Les prélèvements d'autopsie nécessaires à la bonne exécution des expertises toxicologiques. J. Med. Lég. Droit. Med. 1998 ; 41 : 223-233.

8. Litovitz T.L. Clinical manifestations of toxicity in series of 784 boric acid ingestion. Am. J. Emerg. Med. 1988 ; $6: 209-213$.

9. Testud F. Pathologie Toxique Professionnelle et Environnementale. ESKA. 2006.

10. www.tiaft.org - TIAFT reference blood concentration list.

11. Kirschbaum B.B., Qxhoolwerth A.C. Acute aluminum toxicity associated with oral citrate and aluminumcontaining antiacids. Am. J. Med .Sci. 1989 ; 297 : 9-11.

12. Russo L.S., Beale G., Sandroni S., Ballinger W.E. Aluminium intoxication in undialysed adults with chronic renal failure. J. Neurol. Neurosurg. Psychiatry $1992 ; 55$ : 697-700.

13. Garry V.F., Good P.F., Manivel J.C., Perl D.P. Investigation of a fatality from nonoccupational aluminum phosphide exposure : measurement of aluminum in tissue and body fluids as a marker of exposure. J. Lab. Clin. Med. 1993 ; 122 : 739-47. 